\title{
RECURRENCE RATES FOR LOOSELY MARKOV DYNAMICAL SYSTEMS
}

\author{
MARIUSZ URBAŃSKI
}

(Received 19 January 2004; revised 6 June 2005)

\author{
Communicated by $\mathrm{K}$. Wysocki
}

\begin{abstract}
The concept of loosely Markov dynamical systems is introduced. We show that for these systems the recurrence rates and pointwise dimensions coincide. The systems generated by hyperbolic exponential maps, arbitrary rational functions of the Riemann sphere, and measurable dynamical systems generated by infinite conformal iterated function systems are all checked to be loosely Markov.
\end{abstract}

2000 Mathematics subject classification: primary 37C45; secondary 37C40, 37F35, 37A25.

\section{Introduction and preliminaries}

Let $(X, \rho)$ be a metric space, $\mu$ be a Borel probability measure on $X$ and let $T: X \rightarrow X$ be a $\mu$-invariant measurable map. Given $x \in X$, one defines lower and upper pointwise dimensions of $\mu$ at the point $x$ respectively as follows.

$$
\underline{d}_{\mu}(x)=\liminf _{r \rightarrow 0} \frac{\log \mu(B(x, r))}{\log r} \text { and } \bar{d}_{\mu}(x)=\limsup _{r \rightarrow 0} \frac{\log \mu(B(x, r))}{\log r} .
$$

Given in addition $r>0$, one defines

$$
\tau_{r}(x)=\inf \left\{n \geq 1: T^{n}(x) \in B(x, r)\right\}=\inf \left\{n \geq 1: \rho\left(T^{n}(x), x\right)<r\right\} .
$$

The lower and upper recurrence rates of $x$ are defined as

$$
\underline{R}(x)=\liminf _{r \rightarrow 0} \frac{\log \tau_{r}(x)}{-\log r} \text { and } \bar{R}(x)=\limsup _{r \rightarrow 0} \frac{\log \tau_{r}(x)}{-\log r} .
$$

(C) 2007 Australian Mathematical Society $1446-7887 / 07 \$ A 2.00+0.00$ 
Let $\operatorname{HD}(\mu)$ be the Hausdorff dimension of the measure $\mu(\inf \{\operatorname{HD}(Y)\}$ taken over all Borel sets $Y$ with $\mu(Y)=1$ ). It is well-known that if $X$ is a subset of a Euclidean space and $\rho$ is the corresponding Euclidean metric, then $\operatorname{HD}(\mu)=\operatorname{ess} \sup \left(\underline{d}_{\mu}\right)$. The pointwise dimensions and recurrence rates seem to be a priori absolutely unrelated. The former are purely geometric notions whereas the latter are rather dynamical. However Boshernitzan, in his pioneering work [4], has been able to prove the remarkable inequality $\underline{R}(x) \leq \mathrm{HD}(\mu)$ for $\mu$-almost-everywhere $x \in X$. Since then, a number of papers devoted to finding closer relations (under various additional assumptions) between recurrence rates and dimensions have appeared. In the remarkable paper [2], the authors provided a sufficient condition for $\underline{R}(x)=\underline{d}_{\mu}(x)$ and $\bar{R}(x)=\bar{d}_{\mu}(x)$ to hold $\mu$-almost-everywhere. Their condition involved a uniform expanding property and a strong mixing property with respect to the reference partition. We provide a sufficient condition for these two equalities to hold. However in contrast to [2], we do not assume any expanding property and instead of assuming a mixing condition with respect to a reference partition, we impose an assumption on the rate of convergence of the Perron-Frobenius operator associated to the measure $\mu$. We do not have to, therefore, care too much about a good partition, and in particular, about mixing with respect to such a partition. Our general condition, Theorem 2.1, applies to dynamical systems such as hyperbolic exponential maps, arbitrary rational functions of the Riemann sphere and measurable dynamical systems generated by infinite conformal iterated function systems. Let us now introduce and describe some technical notions and results needed in the proof of Theorem 2.1.

Given two points $x \in X$ and $y \in B(x, r)$, the return time of the point $y$ into $X$ is defined as $\tau_{r}(y, x)=\min \left\{k \geq 1: \rho\left(T^{k}(y), x\right)<r\right\}$. For each $x \in X$ and all $r, \varepsilon>0$, consider the set

$$
A_{\varepsilon}(x, r)=\left\{y \in B(x, r): \tau_{r}(y, x) \leq \mu(B(x, r))^{-1+\varepsilon}\right\} .
$$

The measure $\mu$ is said to have long return time with respect to $T$ if

$$
\liminf _{r \rightarrow 0} \frac{\log \mu\left(A_{\varepsilon}(x, r)\right)}{\log \mu(B(x, r))}>1
$$

for $\mu$-almost-everywhere $x \in X$ and all $\varepsilon>0$ sufficiently small. The following fact has been proved in [1].

PROPOSITION 1.1. Let $X$ be a subset of a Euclidean space, $\mu$ be a Borel probability measure on $X$ and let $T: X \rightarrow X$ be a $\mu$-invariant measurable map. If $\mu$ has long return time and $\underline{d}_{\mu}(x)>0$ for $\mu$-almost-everywhere $x \in X$, then $\underline{d}_{\mu}(x)=\underline{R}(x)$ and $\bar{d}_{\mu}(x)=\bar{R}(x)$ for $\mu$-almost-everywhere $x \in X$.

A Borel probability measure on a Euclidean space $\mathbb{R}^{d}$ is said to be weakly diametrically regular if for $\mu$-almost-everywhere $x \in \mathbb{R}^{d}$ and every $\varepsilon>0$, there exists 
$\delta>0$ such that if $r<\delta$, then $\mu(B(x, 2 r)) \leq \mu(B(x, r)) r^{-\varepsilon}$. The following result also comes from [1].

PROPOSITION 1.2. Every Borel probability measure on a Euclidean space is weakly diametrically regular.

The return time $\tau(A)$ of a set $A$ into itself is defined as

$$
\tau(A)=\min \left\{n \geq 1: T^{n}(A) \cap A \neq \emptyset\right\} .
$$

Given a partition $\alpha$ of $X$ and a point $x \in X$, denote by $\alpha(x)$ the only element of $\alpha$ containing $x$. Given in addition an integer $n \geq 1$, the refined partition,

$$
\alpha \vee T^{-1}(\alpha) \vee \cdots \vee T^{n-1}(\alpha)
$$

is denoted by $\alpha_{n}$. The basic property joining the notions above is captured by the following result, proven in [14].

PROPOSITION 1.3. Suppose that $T: Y \rightarrow Y$ is a measurable transformation preserving an ergodic probability measure $\mu$. If $\alpha$ is a finite or countable partition of $Y$ with positive entropy $\mathrm{h}_{\mu}(T, \alpha)$, then $\liminf _{n \rightarrow \infty} \frac{1}{n} \tau\left(\alpha_{n}(x)\right) \geq 1$ for $\mu$-almosteverywhere $x \in Y$.

\section{Loosely Markov maps}

Let $(X, \rho)$ be a metric space and let $f: X \rightarrow X$ be a Borel map that is at most countable-to-one. Let $\mu$ be a Borel probability $f$-invariant measure on $X$. Suppose that $f$ is non-singular with respect to $\mu$, that is, $\mu(f(A))=0$ if $\mu(A)=0$. Consider $J_{\mu}$, the Jacobian of the map $f$ with respect to the measure $\mu$. The PerronFrobenius operator $\mathcal{L}_{\mu}$ of $f$ with respect to the measure is given by the formula

$$
\mathcal{L}_{\mu}(g)(x)=\sum_{y \in f^{-1}(x)} J_{\mu}^{-1}(y) g(y) .
$$

Since the measure $\mu$ is $f$-invariant, $\mathcal{L}_{\mu}(\mathbf{1})=1$. Suppose that $J_{\mu}: X \rightarrow(0,+\infty)$ is a bounded locally Hölder continuous function, with an exponent $\xi>0$. Denote the class of all such functions by $\mathrm{H}_{\xi}$. Let $C_{b}(X)$ denote the Banach space of all bounded real-valued functions on $X$ endowed with the supremum norm $\|\cdot\|$. Then $\mathrm{H}_{\xi}$ becomes a Banach space when equipped with the norm $\|\cdot\|_{\xi}$ defined as $\|g\|_{\xi}=v_{\xi}(g)+\|g\|_{\infty}$, where

$$
v_{\xi}(g)=\sup \left\{\frac{|g(y)-g(x)|}{\rho^{\xi}(y, x)}: x \in X, y \in B(x, \delta) \backslash\{x\}\right\}
$$


and $\delta$ is small enough.

Suppose that the Perron-Frobenius operator $\mathcal{L}_{\mu}$ preserves $C_{b}(X)$ and $\mathrm{H}_{\xi}$. Assuming from now on that $X$ is Borel subset of a Euclidean space and $\rho$ is the standard Euclidean metric, the dynamical system $(f, \mu)$ is said to be loosely Markov provided that the following conditions are satisfied. There exists a continuous function $C_{1}: X \rightarrow$ $(0,+\infty)$ such that

(a) There exists $\gamma \in(0,1)$ such that for all $g \in \mathrm{H}_{\xi}, n \geq 0$, and $x \in X$

$$
\left|\mathcal{L}_{\mu}^{n}(g)(x)-\mu(g)\right| \leq C_{1}(x) \gamma^{n}\|g\|_{\xi} .
$$

(b) For $\mu$-almost-everywhere $x \in X$ there exists $\chi(x)>0$ and a countable partition $\alpha$ of $X$ (by Borel sets) such that $\mathrm{h}_{\mu}(f, \alpha)>0$ and $\alpha_{n}(x) \supset B(x, \exp (-\chi(x) n))$ for $\mu$-almost-everywhere $x \in X$ and all $n \geq 0$ large enough.

(c) For $\mu$-almost-everywhere $x \in X, 0<\underline{d}_{\mu}(x) \leq \bar{d}_{\mu}(x)<\infty$.

We shall now prove a general theorem (which in some sense corresponds to [2, Theorem 6]) such that all the concluding results stated in the next sections follow from it after some appropriate preparations.

THEOREM 2.1. If $(f, \mu)$ is a loosely Markov dynamical system, then $\underline{R}(x)=\underline{d}_{\mu}(x)$ and $\bar{R}(x)=\bar{d}_{\mu}(x)$ for $\mu$-almost-everywhere $x \in X$.

Proof. Fix a Borel set $W \subset X$ such that $\mu(W)=1$ and conditions (b) and (c) are satisfied for all $x \in W$. Combining condition (b) and Proposition 1.3, we see that

$$
\liminf _{r \rightarrow 0} \frac{\tau(B(x, r))}{-\log r}=\liminf _{n \rightarrow \infty} \frac{\tau(B(x, \exp (-\chi(x) n)))}{\chi(x) n} \geq \liminf _{n \rightarrow \infty} \frac{\tau\left(\alpha_{n}(x)\right)}{\chi(x) n} \geq \frac{1}{\chi(x)} .
$$

This implies that

$$
B(x, r) \cap f^{-k}(B(x, r))=\emptyset
$$

for all $r>0$ small enough and all $k \leq-(1 / 2 \chi) \log r$, where $\chi:=\chi(x)$. It follows from condition (c) that for every $s>0$ sufficiently small (depending on $x$ ), we have

$$
s^{2 \bar{d}_{\mu}(x)} \leq \mu(B(x, s)) \leq s^{d_{\mu}(x) / 2} .
$$

Let $B_{r}=B(x, r)$ for all $r \in(0,1)$. Given in addition $\kappa>0$, define the function $\zeta_{r, k}:[0,+\infty) \rightarrow[0,1]$ by the following formula.

$$
\zeta_{r, \kappa}(t)= \begin{cases}1 & \text { if } 0 \leq t \leq r \\ r^{-\kappa}\left(r+r^{\kappa}-t\right) & \text { if } r \leq t \leq r+r^{\kappa} \\ 0 & \text { if } t \geq r+r^{\kappa}\end{cases}
$$


Define $g_{r, k}: X \rightarrow[0,1]$ by setting $g_{r, k}(z)=\zeta_{r, k}(\rho(z, x))$. Since the function $z \mapsto \rho(z, x)$ is Lipschitz continuous with Lipschitz constant equal to 1 , and since $\zeta_{r, k}$ is Lipschitz continuous with Lipschitz constant equal to $r^{-\kappa}$, the composite function $g_{r, \kappa}: X \rightarrow[0,1]$ is Lipschitz continuous with Lipschitz constant equal to $r^{-\kappa}$. In particular, $g_{r, \kappa} \in \mathrm{H}_{\xi}$ and $\left\|g_{r, \kappa}\right\|_{\xi} \leq r^{-\kappa}$. We now show that the measure $\mu$ has long return time at the point $x$. Since $C_{1}: X \rightarrow(0,+\infty)$ is continuous, we may assume $r>0$ to be so small that that $C_{1}(z) \leq 2 C_{1}(x)$ for all $z \in B_{r}$. It follows from condition (a) of the loosely Markov property and Proposition 1.2 that for every $k \geq 0$

$$
\begin{aligned}
\mu\left(B(x, r) \cap f^{-k}(B(x, r))\right) & \leq \int_{B_{r}} \mathcal{L}_{\mu}^{k}\left(\mathbf{1}_{B_{r}}\right) d \mu \leq \int_{B_{r}} \mathcal{L}_{\mu}^{k}\left(g_{r, k}\right) d \mu \\
& \leq \int_{B_{r}}\left(\mu\left(g_{r, k}\right)+C_{1} \gamma^{k}\left\|g_{r, k}\right\|_{\xi}\right) d \mu \\
& \leq \int_{B_{r}}\left(\mu\left(g_{r, k}\right)+2 C_{1}(x) r^{-\kappa} \gamma^{k}\right) d \mu .
\end{aligned}
$$

Assuming that $r \in(0,1)$ is sufficiently small, using $(2.2)$, and with $\beta=-(\log \gamma) / 4 \chi$ and some universal constant $C_{2}>0$, we have that

$$
r^{-\beta} \sum_{k=-(\log r) / 2 \chi}^{2(\log r) /(\log \gamma)} \gamma^{k} \leq C_{2} r^{-\beta} r^{-(\log \gamma) / 2 \chi}=C_{2} r^{-(\log \gamma) / 4 \chi} \leq C_{2} \mu\left(B_{r}\right)^{-(\log \gamma) / 8 \chi \bar{d}_{\mu}(x)}
$$

and

$$
\begin{aligned}
\mu\left(g_{r, \beta}\right) & \leq \mu\left(B_{r+r^{\beta}}\right) \leq \mu\left(B_{2 r_{\min \{1 \beta \beta\}}}\right) \leq 2^{d_{\mu}(x) / 2} r^{\underline{d}_{\mu}(x) \min \{1, \beta] / 2} \\
& \leq 2^{\underline{d}_{\mu}(x) / 2} \mu\left(B_{r}\right)^{d_{\mu}(x) \min (1, \beta\} / 4 \bar{d}_{\mu}(x)} .
\end{aligned}
$$

Combining these two last formulas, (2.3) and (2.2), we obtain

$$
\begin{aligned}
& \sum_{k=-(\log r) / 2 x}^{2(\log r) /(\log \gamma)} \mu\left(B_{r} \cap f^{-k}\left(B_{r}\right)\right) \\
& \leq \sum_{k=-(\log r) / 2 x}^{2(\log r) /(\log \gamma)} \int_{B_{r}}\left(\mu\left(g_{r, \beta}\right)+2 C_{1}(x) r^{-\beta} \gamma^{k}\right) d \mu \\
& \leq\left(\left(\frac{2}{\log \gamma}+\frac{1}{2 \chi}\right) \log (r) \mu\left(g_{r, \beta}\right)+2 C_{1}(x) r^{-\beta} \sum_{k=-(\log r) / 2 x}^{2(\log r) /(\log \gamma)} \gamma^{k}\right) \mu\left(B_{r}\right) \\
& \leq\left(\left(\frac{2}{\log \gamma}+\frac{1}{2 \chi}\right) \log (r) 2^{d_{\mu}(x) / 2} \mu\left(B_{r}\right)^{d_{\mu}(x) \min (1, \beta) / 4 \bar{d}_{\mu}(x)}\right. \\
& \left.\quad+2 C_{1}(x) C_{2} \mu\left(B_{r}\right)^{-(\log \gamma) / 8 x \bar{d}_{\mu}(x)}\right) \mu\left(B_{r}\right)
\end{aligned}
$$




$$
\begin{aligned}
& \leq\left(\mu\left(B_{r}\right)^{d_{\mu}(x) \min \{1, \beta\} / 8 \vec{d}_{\mu}(x)}+2 C_{1}(x) C_{2} \mu\left(B_{r}\right)^{-(\log \gamma) / 8 x \vec{d}_{\mu}(x)}\right) \mu\left(B_{r}\right) \\
& \leq\left(1+2 C_{1}(x) C_{2}\right) \mu\left(B_{r}\right)^{1+\theta}
\end{aligned}
$$

where $\theta=\left(1 / 8 \bar{d}_{\mu}(x)\right) \min \left\{d_{\mu}(x) \min \{1, \beta\},-(\log \gamma) / \chi\right\}$. Using (2.2) we also have that

$$
\begin{aligned}
r^{-1} \sum_{k=2(\log r) /(\log \gamma)}^{\mu\left(B_{r}\right)^{-1+\varepsilon}} \gamma^{k} \leq C_{3} r^{-1} \gamma^{2(\log r) /(\log \gamma)} & =C_{3} r^{-1} \exp \left(2 \frac{\log r \log \gamma}{\log \gamma}\right) \\
& =C_{3} r \leq C_{3} \mu\left(B_{r}\right)^{1 / 2 \bar{d}_{\mu}(x)}
\end{aligned}
$$

with some universal constant $C_{3}>0$. Applying (2.3) and Proposition 1.2, we thus get, for all $r>0$ small enough, that

$$
\begin{aligned}
& \sum_{k=2(\log r) /(\log \gamma)}^{\mu\left(B_{r}\right)^{-1+\varepsilon}} \mu\left(B_{r} \cap f^{-k}\left(B_{r}\right)\right) \\
& \quad \leq \sum_{k=2(\log r) /(\log \gamma)}^{\mu\left(B_{r}\right)^{-1+\varepsilon}} \int_{B_{r}}\left(\mu\left(g_{r, 1}\right)+2 C_{1}(x) r^{-1} \gamma^{k}\right) d \mu \\
& \quad \leq\left(\mu\left(B_{r}\right)^{-1+\varepsilon} \mu\left(g_{r, 1}\right)+2 C_{1}(x) r^{-1} \sum_{k=2(\log r) /(\log \gamma)}^{\mu\left(B_{r}\right)^{-1+\varepsilon}}\right) \mu\left(B_{r}\right) \\
& \quad \leq\left(\mu\left(B_{r}\right)^{-1+\varepsilon} \mu\left(B_{2 r}\right)+2 C_{1}(x) C_{3} \mu\left(B_{r}\right)^{1 / 2 \bar{d}_{\mu}(x)}\right) \mu\left(B_{r}\right) \\
& \quad=\left(\mu\left(B_{r}\right)^{-1+\varepsilon} \mu\left(B_{r}\right) r^{-\varepsilon / 2}+2 C_{1}(x) C_{3} \mu\left(B_{r}\right)^{1 / 2 \bar{d}_{\mu}(x)}\right) \mu\left(B_{r}\right) \\
& \quad=\left(\mu\left(B_{r}\right) r^{\varepsilon / 2}+2 C_{1}(x) C_{3} \mu\left(B_{r}\right)^{1 / 2 \bar{d}_{\mu}(x)}\right) \mu\left(B_{r}\right) \\
& \leq\left(1+2 C_{1}(x) C_{3}\right) \mu\left(B_{r}\right)^{1+\eta},
\end{aligned}
$$

where $\eta=\min \left\{\varepsilon, 1 / \bar{d}_{\mu}(x)\right\} / 2$. Combining this with (1.1), (2.1) and (2.4), we obtain for all $r \in(0,1)$ sufficiently small that

$$
\begin{aligned}
\mu\left(A_{\varepsilon}(x, r)\right) & \leq \sum_{k=0}^{\mu\left(B_{r}\right)^{-1+\varepsilon}} \mu\left(B_{r} \cap f^{-k}\left(B_{r}\right)\right) \\
& \leq\left(1+2 C_{1}(x) C_{2}\right) \mu\left(B_{r}\right)^{1+\theta}+\left(1+2 C_{1}(x) C_{3}\right) \mu\left(B_{r}\right)^{1+\eta} \\
& \leq 2 C_{1}(x)\left(1+C_{2}+C_{3}\right) \mu\left(B_{r}\right)^{1+\min \{\theta, \eta\}} .
\end{aligned}
$$

Thus

$$
\frac{\log \left(\mu\left(A_{\varepsilon}(x, r)\right)\right)}{\log \left(\mu\left(B_{r}\right)\right)} \geq \frac{\log \left(2 C_{1}(x)\left(1+C_{2}+C_{3}\right)\right)}{\log \left(\mu\left(B_{r}\right)\right)}+(1+\min \{\theta, \eta\})
$$


and consequently

$$
\liminf _{r \rightarrow 0} \frac{\log \left(\mu\left(A_{\varepsilon}(x, r)\right)\right)}{\log \left(\mu\left(B_{r}\right)\right)} \geq(1+\min \{\theta, \eta\})>1 .
$$

Hence, the measure $\mu$ has long return time and the theorem follows by applying Proposition 1.1.

\section{Rational functions of the Riemann sphere $\mathbb{C}$}

In this short section $f: \overline{\mathbb{C}} \rightarrow \overline{\mathbb{C}}$ is an arbitrary rational function of degree $\geq 2$. Denote by $J(f)$ the Julia set of the function $f$. A function $\phi: J(f) \rightarrow \mathbb{R}$ is assumed to be Hölder continuous and to satisfy inequality $\mathrm{P}(\phi)>\sup (\phi)$, where $\mathrm{P}(\phi)$ is the topological pressure of $\phi$ (see [12] and [13] for its definition and a fairly thorough exposition of its properties). It was shown in [5] that in this situation there exists a unique equilibrium state (see also [12] and [13]) $\mu_{\phi}$ of $\phi$ and $f: J(f) \rightarrow J(f)$. Our aim is to show that the dynamical system $\left(J(f), \mu_{\phi}\right)$ is loosely Markov. Indeed, it was proved in [12] (see Theorem 9.4.2) that

$$
\underline{d}_{\mu_{\phi}}(x)=\bar{d}_{\mu_{\phi}}(x)=\mathrm{HD}\left(\mu_{\phi}\right)=\frac{\mathbf{h}_{\mu_{\phi}}}{\chi_{\mu_{\phi}}}
$$

for $\mu_{\phi}$-almost-everywhere $x \in J(f)$. In particular, condition (c) of the definition of a loosely Markov map is satisfied. Condition (b) was established in the proof of Theorem 9.4.2 from [12]. Finally, condition (a) is exactly the content of Theorem 10 in [8]. Hence, applying Theorem 2.1 and using (3.1), we get the following.

THEOREM 3.1. If $f: \overline{\mathbb{C}} \rightarrow \overline{\mathbb{C}}$ is a rational function of degree $\geq 2$ and if $\phi:$ $J(f) \rightarrow \mathbb{R}$ is a Hölder continuous potential such that $\mathrm{P}(\phi)>\sup (\phi)$, then for $\mu_{\phi}$-almost-everywhere $z \in J(f)$, the number $R_{\mu_{\phi}}(z)$ exists and moreover

$$
R_{\mu_{\phi}}(z)=\mathrm{HD}\left(\mu_{\phi}\right)=\frac{\mathrm{h}_{\mu_{\phi}}}{\chi_{\mu_{\phi}}} .
$$

\section{Hyperbolic exponential maps}

In this section we consider the maps $f_{\lambda}: \mathbb{C} \rightarrow \mathbb{C}, \lambda \in \mathbb{C} \backslash\{0\}$, given by the formula

$$
f_{\lambda}(z)=\lambda e^{z}
$$

We assume that $f_{\lambda}$ is hyperbolic, that is, $f_{\lambda}$ has an attracting periodic cycle (this is always the case if $\lambda \in \mathbb{C}$ is in a sufficiently small neighborhood of the interval $(0,1 / e)$ ). 
We are interested in the recurrence rates for the mapping $F_{\lambda}$, canonically associated with $f_{\lambda}$, which carries essentially all significant information about the dynamics of $f_{\lambda}$ and is more suitable for measure theoretic considerations. So, the equivalence relation ' $\sim$ ' on $\mathbb{C} \times \mathbb{C}$ is defined as follows: $z \sim w$ if and only if $w-z \in 2 \pi i \mathbb{Z}$. The map $f_{\lambda}$ projects down to the holomorphic map $F_{\lambda}$ of the cylinder $Q=\mathbb{C} / \sim$ such that if $\Pi: \mathbb{C} \rightarrow Q$ denotes the canonical projection, then $f_{\lambda} \circ \Pi=\Pi \circ F_{\lambda}$. From now on we will drop the subscript $\lambda$ and simply write $f$ and $F$. Let

$$
\delta=\frac{1}{2} \min \left\{\frac{1}{2}, \operatorname{dist}\left(J(F),\left\{F^{n}(\Pi(0)): n \geq 0\right\}\right)\right\}
$$

Since the map $f: J(f) \rightarrow J(f)$ is hyperbolic, we see that $\delta>0$. For every $n \geq 1$ and every $v \in J(F)$ the map $F_{v}^{-n}: B\left(F^{n}(v), 2 \delta\right) \rightarrow P$ is then defined to be the holomorphic inverse branch of $F^{n}$ on $B\left(F^{n}(v), 2 \delta\right)$, sending $F^{n}(v)$ to $v$. Let $\phi$ be a real-valued Hölder continuous function defined on some Euclidean $R$-neighbourhood of the Julia set $J(F) \subset \mathbb{C}, R \in(0, \delta / 2)$. Hölder continuous means here that there exists an $\alpha>0$ and for all $r \in(0, R)$, there exists $H_{r}>0$ such that if $|y-x| \leq r$ then $|\phi(y)-\phi(x)| \leq H_{r}|y-x|^{\alpha}$. One can prove (see [15]) that for every $z \in J(F)$ the following limit exists and is independent of the point $z$.

$$
\mathrm{P}(\phi)=\lim _{n \rightarrow \infty} \frac{1}{n} \log \sum_{x \in F^{-n}(z)} \exp \left(S_{n} \phi(x)\right) .
$$

The number $\mathrm{P}(\phi)$ is called the topological pressure of the potential $\phi$. The following easy technical fact is also established in [15].

LEMMA 4.1. For every $\alpha$-Hölder function $\phi: J(F) \rightarrow \mathbb{C}$ there exists a constant $L_{\phi}>0$ such that $\left|S_{n} \phi\left(F_{v}^{-n}(y)\right)-S_{n} \phi\left(F_{v}^{-n}(x)\right)\right| \leq L_{\phi}|y-x|^{\alpha}$ for all $n \geq 1$, all $x, y \in J(F)$ with $|x-y| \leq \delta$ and all $v \in F^{-n}(x)$. In particular,

$$
\exp \left(S_{n} \phi\left(F_{v}^{-n}(y)\right)\right) \leq T \exp \left(S_{n} \phi\left(F_{v}^{-n}(x)\right)\right),
$$

where $T=\exp \left(L \delta^{\alpha}\right)$.

A Hölder continuous function $\phi: J(F) \rightarrow \mathbb{R}$ is called $1^{+}$-tame if there exists $\kappa>1$ such that $A_{\phi}:=\sup \{|\phi(z)+\kappa \operatorname{Re} z|: z \in J(F)\}<+\infty$. The following three basic facts have been proved in [15].

THEOREM 4.2. If $f: \mathbb{C} \rightarrow \mathbb{C}$ is hyperbolic and $\phi: J(F) \rightarrow \mathbb{R}$ is a $1^{+}$-tame potential, then there exists a unique Borel probability measure $m_{\phi}$ on $J(F)$ such that

$$
m_{\phi}\left(F_{v}^{-n}(B(z, \delta))\right)=\int_{B(z, \delta)} \exp \left(S_{n} \phi\left(F_{v}^{-n}(w)\right)-\mathrm{P}(\phi) n\right) d m_{\phi}(w)
$$

for all $n \geq 1$, all $z \in J(F)$ and all $v \in F^{-n}(z)$. 
THEOREM 4.3. There exists a unique Borel probability F-invariant measure $\mu_{\phi}$ absolutely continuous with respect to $m_{\phi}$. In addition, $\mu_{\phi}$ is ergodic, equivalent to $m_{\phi}$ and the Radon-Nikodym derivative $d \mu_{\phi} / d m_{\phi}$ has a continuous everywhere (on $J(F)$ ) positive and uniformly bounded version.

THEOREM 4.4. The invariant measure $\mu_{\phi}$ is an equilibrium state of the potential $\phi$, that is $\mathrm{P}(\phi)=\mathrm{h}_{\mu_{\phi}}+\int \phi d \mu_{\phi}$.

The following two more technical facts have also been proved in [15].

THEOREM 4.5. The entropy $\mathrm{h}_{\mu_{\phi}}(F)$ is positive.

THEOREM 4.6. The Lyapunov exponent $\chi_{\mu_{\phi}}=\int \log \left|F^{\prime}\right| d \mu_{\phi}$ is finite.

We verify that the dynamical system $\left(F, \mu_{F}\right)$ is loosely Markov starting with the following.

THEOREM 4.7. If $f: \mathbb{C} \rightarrow \mathbb{C}$ is a hyperbolic exponential map and $\phi: J(F) \rightarrow \mathbb{R}$ is a $1^{+}$-tame potential, then for $\mu_{\phi}$-almost-everywhere $z \in J(F)$, the local dimension $d_{\mu_{\phi}}(z)$ exists and is equal to $\mathrm{h}_{\mu_{\phi}} / \chi_{\mu_{\phi}}$. In particular, $\operatorname{HD}\left(\mu_{\phi}\right)=\mathbf{h}_{\mu_{\phi}} / \chi_{\mu_{\phi}}$.

PROOF. In view of Birkhoff's ergodic theorem, there exists a Borel set $X \subset J(F)$ such that $\mu_{\phi}(X)=1$ and, for every $x \in X$,

$$
\lim _{n \rightarrow \infty} \frac{1}{n} \log \left|\left(F^{n}\right)^{\prime}(x)\right|=\chi_{\mu_{\diamond}} \text { and } \lim _{n \rightarrow \infty} \frac{1}{n} S_{n} \phi(x)=\int \phi d \mu_{\phi} .
$$

Fix $x \in X$ and $\varepsilon>0$. There exists $k \geq 1$ such that, for every $n \geq k$,

$$
\left|\frac{1}{n} \log \right|\left(F^{n}\right)^{\prime}(x)\left|-\chi_{\mu_{\star}}\right|<\varepsilon .
$$

Fix $r \in(0, \delta)$ and let $n=n(r) \geq 0$ be the largest integer such that

$$
B(x, r) \subset F_{x}^{-n}\left(B\left(F^{n}(x), \delta\right)\right) .
$$

Then $B(x, r)$ is not contained in $F_{x}^{-(n+1)}\left(B\left(F^{n+1}(x), \delta\right)\right)$ and it follows from the 1/4-Koebe's distortion theorem that

$$
r \geq \delta\left|\left(F^{n+1}\right)^{\prime}(x)\right|^{-1} / 4
$$


Taking $r>0$ sufficiently small, we may assume that $n \geq k$. Applying Theorem 4.2 and using (4.3) along with Lemma 4.1, we get that

$$
\begin{aligned}
m_{\phi}(B(x, r)) & \leq \int_{B\left(F^{n}(x), \delta\right)} \exp \left(S_{n} \phi \circ F_{x}^{-n}-\mathrm{P}(\phi) n\right) d m_{\phi} \\
& \leq T \exp \left(S_{n} \phi(x)-\mathrm{P}(\phi) n\right) m_{\phi}\left(B\left(F^{n}(x), \delta\right)\right) \\
& \leq T \exp \left(S_{n} \phi(x)-\mathrm{P}(\phi) n\right) .
\end{aligned}
$$

Applying now (4.4) and (4.2), we obtain

$$
\begin{aligned}
\frac{\log m_{\phi}(B(x, r))}{\log r} & \geq \frac{\log T+S_{n} \phi(x)-\mathrm{P}(\phi) n}{\log r} \\
& \geq \frac{\log T+S_{n} \phi(x)-\mathrm{P}(\phi) n}{\log \delta-\log 4-\log \left|\left(F^{n+1}\right)^{\prime}(x)\right|} \\
& \geq \frac{\log T+S_{n} \phi(x)-\mathrm{P}(\phi) n}{\log \delta-\log 4-\left(\chi_{\mu_{\phi}}-\varepsilon\right)(n+1)} .
\end{aligned}
$$

Dividing the numerator and denominator of the last quotient by $n=n(r)$, letting $r \searrow 0$ (which implies that $n(r) \rightarrow \infty$ ), and using the second part of (4.1), we get that

$$
\underline{d}_{m_{\phi}}(x) \geq \frac{-\int \phi d \mu_{\phi}+\mathrm{P}(\phi)}{\chi_{\mu_{\phi}}} .
$$

Since, by Theorem 4.3, the measures $\mu_{\phi}$ and $m_{\phi}$ are equivalent with positive continuous Radon-Nikodym derivatives, we obtain for all $x \in X$ that

$$
\underline{d}_{\mu_{\phi}}(x) \geq \frac{-\int \phi d \mu_{\phi}+\mathrm{P}(\phi)}{\chi_{\mu_{\phi}}} .
$$

For every $s>0$, let $J_{s}=\{z \in J(F):|\operatorname{Re}(z)| \leq s\}$. Take $M$ so large that $\mu_{\phi}\left(J_{M}\right)>0$. Since the measure $m_{\phi}$ is positive on non-empty open subsets of $J(F)$, we get that

$$
W:=\inf \left\{m_{\phi}\left(B(z, \delta): z \in J_{M}\right\}>0 .\right.
$$

In view of ergodicity of the measure $\mu_{\phi}$ and Birkhoff's ergodic theorem, there exists a Borel set $Y \subset X$ such that $\mu_{\phi}(Y)=1$ and

$$
\lim _{n \rightarrow \infty} \frac{1}{n} S_{n}\left(\mathbf{1}_{J_{M}}\right)(x)=\mu_{\phi}\left(J_{M}\right)>0
$$

for all $x \in Y$. In particular, if $\left\{n_{j}\right\}_{j=1}^{\infty}$ is the unbounded, increasing sequence of all integers $n \geq 1$ such that $F^{n}(x) \in J_{M}$, then

$$
\lim _{j \rightarrow \infty} \frac{n_{j+1}}{n_{j}}=1 \text {. }
$$


Keep $x \in Y$ and let $l \geq 0$ be the least integer such that

$$
B(x, r) \supset F_{x}^{-i}\left(B\left(F^{i}(x), \delta\right)\right)
$$

for all $i \geq l$. Taking $r>0$ small enough, we may assume that $l>\max \left\{k, n_{1}\right\}$. Then there exists a unique $j \geq 2$ such that

$$
n_{j-1}<l \leq n_{j} .
$$

Also $F_{x}^{-(l-1)}\left(B\left(F^{l-1}(x), \delta\right)\right)$ is not contained in $B(x, r)$, and it therefore follows from Koebe's distortion theorem that

$$
r \leq K \delta\left|\left(F^{1-1}\right)^{\prime}(x)\right|^{-1} .
$$

It follows from the definition of $l$, formula (4.7), and Lemma 4.1 that

$$
\begin{aligned}
m_{\phi}(B(x, r)) & \geq m_{\phi}\left(F_{x}^{-n_{j}}\left(B\left(F^{n_{j}}(x), \delta\right)\right)\right) \\
& =\int_{B\left(F^{n_{j}}(x), \delta\right)} \exp \left(S_{n_{j}} \phi \circ F_{x}^{-n_{j}}-\mathrm{P}(\phi) n_{j}\right) d m_{\phi} \\
& \geq T^{-1} \exp \left(S_{n_{j}} \phi(x)-\mathrm{P}(\phi) n_{j}\right) m_{\phi}\left(B\left(F^{n_{j}}(x), \delta\right)\right) \\
& \geq W T^{-1} \exp \left(S_{n_{j}} \phi(x)-\mathrm{P}(\phi) n_{j}\right) .
\end{aligned}
$$

Applying (4.2), (4.4) and (4.7), we obtain

$$
\begin{aligned}
\frac{\log m_{\phi}(B(x, r))}{\log r} & \leq \frac{\log W-\log T+S_{n_{j}} \phi(x)-\mathrm{P}(\phi) n_{j}}{\log r} \\
& \leq \frac{\log W-\log T+S_{n} \phi(x)-\mathrm{P}(\phi) n_{j}}{\log (K \delta)-\log \left|\left(F^{l-1}\right)^{\prime}(x)\right|} \\
& \leq \frac{\log W-\log T+S_{n} \phi(x)-\mathrm{P}(\phi) n_{j}}{\log (K \delta)-\left(\chi_{\mu_{\phi}}+\varepsilon\right)(l-1)} \\
& \leq \frac{\log W-\log T+S_{n_{j}} \phi(x)-\mathrm{P}(\phi) n_{j}}{\log (K \delta)-\left(\chi_{\mu_{\phi}}+\varepsilon\right) n_{j-1}} .
\end{aligned}
$$

Dividing numerator and the denominator of the last quotient by $n_{j-1}$, letting $r \searrow 0$ (which implies that $n_{j-1} \rightarrow \infty$ ), and using the second part of (4.1) along with (4.6), we find that

$$
\bar{d}_{m_{\phi}}(x) \leq \frac{-\int \phi d \mu_{\phi}+\mathrm{P}(\phi)}{\chi_{\mu_{\phi}}} .
$$

Since, by Theorem 4.3 , the measures $\mu_{\phi}$ and $m_{\phi}$ are equivalent with positive continuous Radon-Nikodym derivatives, we obtain for all $x \in Y$ that

$$
\bar{d}_{\mu_{\phi}}(x) \leq \frac{-\int \phi d \mu_{\phi}+\mathrm{P}(\phi)}{\chi_{\mu_{\phi}}} \text {. }
$$


Since, by Theorem 4.4, $\mathrm{P}(\phi)-\int \phi d \mu_{\phi}=\mathrm{h}_{\mu_{\phi}}$, combining this inequality with (4.5), completes the proof.

We now prove the existence of a partition with the properties required by condition (b) of the definition of loosely Markov maps.

LEMMA 4.8. If $f: \mathbb{C} \rightarrow \mathbb{C}$ is hyperbolic exponential map and $\phi: J(F) \rightarrow \mathbb{R}$ is a $1^{+}$-tame potential, then there exists a countable partition $\alpha$ of $J(F)$ (consisting of Borel sets) such that $\mathrm{h}_{\mu_{\phi}}(T, \alpha)>0$ and $\alpha_{n}(x) \supset B\left(x, e^{-\kappa(x) n}\right)$ for $\mu_{\phi}$-almost-everywhere $x \in J(F)$, some constant $\chi(x)>0$ and all $n \geq 1$ large enough.

PROOF. It was proven in [17] that if $\mu$ is a Borel probability measure on the cylinder $Q$, then for every $\xi>0$ there exists a partition $\alpha$ of $Q$ with $\operatorname{diam}(\alpha) \leq \xi$ and such that for every $\beta>0$

$$
\sum_{n=0}^{\infty} \sum_{A \in \alpha} \mu\left(B\left(\partial A, e^{-\beta n}\right)\right)<\infty .
$$

Let us apply this fact with $\mu=\mu_{\phi}$. Since $\mathrm{h}_{\mu_{\phi}}>0$, there exists $\xi>0$ so small that $\mathrm{h}_{\mu_{\phi}}(T, \alpha)>0$. Since the measure $\mu_{\phi}$ is $F$-invariant, it follows from (4.9) that

$$
\sum_{n=0}^{\infty} \sum_{A \in \alpha} \mu\left(F^{-n}\left(B\left(\partial A, e^{-\beta n}\right)\right)\right)=\sum_{n=0}^{\infty} \sum_{A \in \alpha} \mu\left(B\left(\partial A, e^{-\beta n}\right)\right)<\infty .
$$

Fix $\varepsilon>0$. It follows from Birkhoff's ergodic theorem, expression (4.10), and the Borel-Cantelli lemma that there exists a Borel set $X_{\varepsilon} \subset J(F)$ such that $\mu_{\phi}\left(X_{\varepsilon}\right)=1$, and for every $x \in X_{\varepsilon}$ there exists $n(x) \geq 1$ such that $F^{n}(x) \notin B\left(\partial A, e^{-\beta n}\right)$ and

$$
\left|\frac{1}{n} \log \right|\left(F^{n}\right)^{\prime}(x)\left|-\chi_{\mu_{\phi}}\right|<\varepsilon
$$

for all $n \geq n(x)$. The former property means that

$$
B\left(F^{n}(x), e^{-\varepsilon n}\right) \subset \alpha\left(F^{n}(x)\right) .
$$

So, fix $x \in X_{\varepsilon}$ and $n \geq n(x)$. There obviously exists $\gamma(x)>0$ so large that

$$
F^{q}\left(B\left(x, e^{-\gamma(x) n}\right)\right) \subset F^{q}\left(B\left(x, e^{-\gamma(x) n(x)}\right)\right) \subset \alpha\left(F^{q}(x)\right) .
$$

for all integers $q=0,1, \ldots, n(x)$. It follows from the 1/4-Koebe's distortion theorem that $F_{x}^{-n}\left(B\left(F^{n}(x), \delta\right)\right) \supset B\left(x, \delta\left|\left(F^{n}\right)^{\prime}(x)\right|^{-1} / 4\right)$. Consequently, all the maps $F^{q}$, $0 \leq q \leq n$, restricted to the ball $B\left(x, \delta\left|\left(F^{n}\right)^{\prime}(x)\right|^{-1} / 4\right)$ are univalent. If $q \in$ $\{n(x), n(x)+1, \ldots, n\}$, then applying (4.11), we get

$$
B\left(x, 8^{-1} \delta \exp \left(-\left(\chi_{\mu_{\phi}}+3 \varepsilon\right) n\right)\right) \subset B\left(x, 8^{-1} \delta\left|\left(F^{n}\right)^{\prime}(x)\right|^{-1}\right),
$$


and it therefore follows from Koebe's distortion theorem that,

$$
\begin{aligned}
F^{q}( & \left.B\left(x, 8^{-1} \delta \exp \left(-\left(\chi_{\mu_{\phi}}+3 \varepsilon\right) n\right)\right)\right) \\
& \subset B\left(F^{q}(x), 8^{-1} \delta K\left|\left(F^{q}\right)^{\prime}(x)\right| \exp \left(-\left(\chi_{\mu_{\phi}}+3 \varepsilon\right) n\right)\right) \\
& \subset B\left(F^{q}(x), 8^{-1} \delta K \exp \left(\left(\chi_{\mu_{\phi}}+\varepsilon\right) q\right) \exp \left(-\left(\chi_{\mu_{\phi}}+3 \varepsilon\right) n\right)\right) \\
& \subset B\left(F^{q}(x), 8^{-1} \delta K \exp \left(\chi_{\mu_{\phi}}(q-n)\right) \exp (-2 \varepsilon n)\right) \\
& \subset B\left(F^{q}(x), 8^{-1} \delta K \exp (-2 \varepsilon n)\right) \\
& \subset B\left(F^{q}(x), \exp (-\varepsilon n)\right) \subset B\left(F^{q}(x), \exp (-\varepsilon q)\right),
\end{aligned}
$$

where the second last inclusion sign was written assuming that $n$ is large enough (depending on $\varepsilon$ ). Since $\chi_{\mu_{\phi}}>0$, we can find $\varepsilon>0$ so small that $\chi_{\mu_{\phi}}+3 \varepsilon \leq 2 \chi_{\mu_{\phi}}$. Then, for all $n \geq n(x)$ large enough, we have that $8^{-1} \delta K \exp \left(-\left(\chi_{\mu_{\phi}}+3 \varepsilon\right) n\right) \geq$ $\exp \left(-3 \chi_{\mu_{\phi}} n\right)$. It follows from (4.14) that

$$
F^{q}\left(B\left(x, \exp \left(-3 \chi_{\mu_{\phi}} n\right)\right)\right) \subset B\left(F^{q}(x), \exp (-\varepsilon q)\right)
$$

for all $n$ large enough and all $q \in\{n(x), n(x)+1, \ldots, n\}$. Combining this with expressions (4.12) and (4.13), we see that $F^{q}\left(B\left(x, e^{-\kappa(x) n}\right)\right) \subset \alpha\left(F^{q}(x)\right)$ for all $n \geq 1$ and all $0 \leq q \leq n$, where $\kappa(x)=\max \left\{\gamma(x), 3 \chi_{\mu_{\phi}}\right\}$. Hence, $B\left(x, e^{-\kappa(x) n}\right) \subset \alpha_{n}(x)$ and we are done.

We are now in position to conclude the main result of this section.

THEOREM 4.9. If $f: \mathbb{C} \rightarrow \mathbb{C}$ is a hyperbolic exponential map and $\phi: J(F) \rightarrow \mathbb{C}$ is a $1^{+}$-tame potential, then for $\mu_{\phi}$-almost-everywhere $z \in J(F), R_{\mu_{\phi}}(z)$ exists and moreover $R_{\mu_{\phi}}(z)=\mathrm{HD}\left(\mu_{\phi}\right)=\mathrm{h}_{\mu_{\phi}} / \chi_{\mu_{\phi}}$.

Proof. We shall verify that the dynamical system $\left(F, \mu_{\phi}\right)$ is loosely Markov. Indeed, condition (a) is guaranteed by appropriate results from [15]. Condition (b) is Lemma 4.8, and condition (c) was established in Theorem 4.7. Thus the proof is completed by applying Theorem 2.1 along with Theorem 4.7 .

\section{Conformal infinite iterated function systems}

Let us first describe the setting of conformal (infinite) iterated function systems introduced in [9]. Let $I$ be a countable index set or alphabet with at least two elements and let $S=\left\{\phi_{i}: X \rightarrow X: i \in I\right\}$ be a collection of injective contractions from a compact metric space $X$ into $X$ for which there exists $0<s<1$ such that $\rho\left(\phi_{i}(x), \phi_{i}(y)\right) \leq s \rho(x, y)$, for every $i \in I$ and for every pair of points $x, y \in X$. Thus, the system $S$ is uniformly contractive. Any such collection $S$ of contractions 
is called an iterated function system. We define the limit set, $J$, of this system as the image of the coding space under a coding map as follows. Let $I^{n}$ denote the space of words of length $n, I^{\infty}$ the space of infinite sequences of symbols in $I, I^{*}=\bigcup_{n \geq 1} I^{n}$ and for $\omega \in I^{n}, n \geq 1$, let $\phi_{\omega}=\phi_{\omega_{1}} \circ \phi_{\omega_{2}} \circ \cdots \circ \phi_{\omega_{n}}$. If $\omega \in I^{*} \cup I^{\infty}$ and $n \geq 1$ does not exceed the length of $\omega$, we denote by $\left.\omega\right|_{n}$ the word $\omega_{1} \omega_{2} \cdots \omega_{n}$. Since given $\omega \in I^{\infty}$, the diameters of the compact sets $\phi_{\left.\omega\right|_{n}}(X), n \geq 1$, converge to zero and since they form a decreasing family, the set $\bigcap_{n=0}^{\infty} \phi_{\left.\omega\right|_{n}}(X)$ is a singleton and therefore, denoting its only element by $\pi(\omega)$, defines the coding map $\pi: I^{\infty} \rightarrow X$. The main object of interest ise the limit set

$$
J=J_{s}=\pi\left(I^{\infty}\right)=\bigcup_{\omega \in I^{\infty}} \bigcap_{n=1}^{\infty} \phi_{\omega \mid n}(X) .
$$

Observe that $J$ satisfies the natural invariance equality, $J=\bigcup_{i \in I} \phi_{i}(J)$. If $I$ is finite, then $J$ is compact and this property fails for infinite systems.

An iterated function system $S=\left\{\phi_{i}: X \rightarrow X: i \in I\right\}$ is said to satisfy the Open Set Condition (OSC) if there exists a nonempty open set $U \subset X$ (in the topology of $X$ ) such that $\phi_{i}(U) \subset U$ for every $i \in I$ and $\phi_{i}(U) \cap \phi_{j}(U)=\emptyset$ for every pair $i, j \in I$, $i \neq j$. We do not exclude the possibility that $\overline{\phi_{i}(U)} \cap \overline{\phi_{j}(U)} \neq \emptyset$.

An iterated function system $S$ satisfying the OSC is said to be conformal if $X \subset \mathbb{R}^{d}$ for some $d \geq 1$ and the following conditions are satisfied.

(1a) $U=\operatorname{Int}_{\mathbb{R}^{d}}(X)$.

(1b) There exists an open connected set $V$ such that $X \subset V \subset \mathbb{R}^{d}$ such that all maps $\phi_{i}, i \in 1$, extend to $C^{1}$ conformal diffeomorphisms of $V$ into $V$. For $d=1$ this just means that all the maps $\phi_{i}, i \in I$, are $C^{1}$ monotone diffeomorphisms, for $d \geq 2$ the words $C^{1}$ conformal mean holomorphic or antiholomorphic, and for $d>2$ the maps $\phi_{i}, i \in I$ are Möbius transformations. The proof of the last statement can be found in [3] for example, where it is called Liouville's theorem.

(1c) There exist $\gamma$ and $l>0$, such that for every $x \in X \subset \mathbb{R}^{d}$ there exists an open cone $\operatorname{Con}(x, \gamma, l) \subset \operatorname{Int}(X)$ with vertex $x$, central angle of Lebesgue measure $\gamma$, and altitude $l$.

(1d) Bounded Distortion Property (BDP). There exists $K \geq 1$ such that

$$
\left|\phi_{\omega}^{\prime}(y)\right| \leq K\left|\phi_{\omega}^{\prime}(x)\right|
$$

for every $\omega \in I^{*}$ and every pair of points $x, y \in V$, where $\left|\phi_{\omega}^{\prime}(x)\right|$ means the norm of the derivative.

Let us now collect some geometric consequences of (BDP). We have for all words $\omega \in I^{*}$ and all convex subsets $C$ of $V$

$$
\operatorname{diam}\left(\phi_{\omega}(C)\right) \leq\left\|\phi_{\omega}^{\prime}\right\| \operatorname{diam}(C) \text { and } \operatorname{diam}\left(\phi_{\omega}(V)\right) \leq D\left\|\phi_{\omega}^{\prime}\right\|,
$$


where the norm $\|\cdot\|$ is the supremum norm taken over $V$ and $D \geq 1$ is a constant depending only on $V$. Moreover,

$$
\operatorname{diam}\left(\phi_{\omega}(X)\right) \geq D^{-1}\left\|\phi_{\omega}^{\prime}\right\| \quad \text { and } \quad \phi_{\omega}(B(x, r)) \supset B\left(\phi_{\omega}(x), K^{-1}\left\|\phi_{\omega}^{\prime}\right\| r\right)
$$

for every $x \in X, 0<r \leq \operatorname{dist}(X, \partial V)$, and every word $\omega \in I^{*}$.

Let $\sigma: I^{\infty} \rightarrow I^{\infty}$ be the shift mapping, that is, cutting off the firs coordinate. In order to define the dynamical systems we want to deal with in this section we first state the following special case of Theorem 4.4.1 proven in [10].

THEOREM 5.1. If $\mu$ is a Borel shift-invariant ergodic probability measure on $I^{\infty}$, then

$$
\mu \circ \pi^{-1}\left(\phi_{\omega}(X) \cap \phi_{\tau}(X)\right)=0
$$

for all incomparable words $\omega, \tau \in I^{*}$.

It follows from this theorem that for $\mu$-almost-everywhere $x \in J$ there exists exactly one element $i \in I$ such that $x \in \phi_{i}(J)$. Setting $f(x)=\phi_{i}^{-1}(x)$, we obtain a dynamical system $f: J \rightarrow J$ defined $\mu$-almost-everywhere on $J$. Given a subset $Y$ of $I^{\infty}$ and $i \in I$, let $[i Y]=\left\{i \omega \in I^{\infty}: \omega \in Y\right\}$. Using shift-invariantness of the measure $\mu$, we get for every Borel set $A \subset J$ that

$$
\begin{aligned}
\mu \circ \pi^{-1}\left(f^{-1}(A)\right) & =\mu \circ \pi^{-1}\left(\bigcup_{i \in I} \phi_{i}(A)\right) \\
& =\mu\left(\bigcup_{i \in I} \pi^{-1}\left(\phi_{i}(A)\right)\right)=\mu\left(\bigcup_{i \in I}\left[i \pi^{-1}(A)\right]\right) \\
& =\mu\left(\sigma^{-1}\left(\pi^{-1}(A)\right)\right)=\mu \circ \pi^{-1}(A) .
\end{aligned}
$$

This means that the measure $\mu \circ \pi^{-1}$ is $f$-invariant and we can consider the metric dynamical system $\left(f, \mu \circ \pi^{-1}\right)$. The Lyapunov characteristic exponent $\chi_{\mu \circ \pi^{-1}}(f)$ of this system is defined by the formula

$$
\chi_{\mu \circ \pi^{-1}}(f)=-\int_{I^{\infty}} \log \left|\phi_{\omega_{1}}^{\prime}(\pi(\sigma(\omega)))\right| d \mu \circ \pi^{-1}(\omega)>0 .
$$

We could have defined this quantity in terms of the dynamical system $\left(f, \mu \circ \pi^{-1}\right)$ as this system and the shift map $(\sigma, \mu)$ are isomorphic via the projection map $\pi: I^{\infty} \rightarrow$ $J$. In order to relate the recurrence rates and pointwise dimensions of the dynamical system $\left(f, \mu \circ \pi^{-1}\right)$, we need to restrict ourselves to a smaller, though fairly large and geometrically significant, class of shift-invariant measures $\mu$ generated by the 
summable Hölder families of functions. Following [6], [10] and [16], we define these families and measures as follows. Fix $\beta>0$ and let $G=\left\{g^{(i)}: X \rightarrow \mathbb{R}: i \in I\right\}$ be a family of continuous functions. For each $n \geq 1$, let

$$
V_{n}(G)=\sup _{\omega \in I^{n}} \sup _{x, y \in X}\left\{\left|g^{\left(\omega_{1}\right)}\left(\phi_{\sigma(\omega)}(x)\right)-g^{\left(\omega_{1}\right)}\left(\phi_{\sigma(\omega)}(y)\right)\right|\right\} \mathrm{e}^{\beta(n-1)},
$$

and assume that $V_{\beta}(G)=\sup _{n \geq 1}\left\{V_{n}(G)\right\}<\infty$. The collection $G$ is then called a Hölder family of functions (of order $\beta$ ). If, in addition, $\sum_{i \in I} \mathrm{e}^{\sup \left(g^{(i)}\right)}<\infty$, then the family $G$ is called a summable Hölder family of functions of order $\beta$. Throughout this section the family $F$ is assumed to be summable Hölder of some order $\beta>0$. Following the classical thermodynamic formalism, we defined the topological pressure of $F$ by setting

$$
\mathrm{P}(G)=\lim _{n \rightarrow \infty} \frac{1}{n} \log \sum_{|\omega|=n} \exp \left(\sup _{x} \sum_{j=1}^{n} g^{\omega_{j}} \circ \phi_{\sigma^{j} \omega}\right) .
$$

The limit indeed exists since the logarithm of the partition function

$$
Z_{n}(G)=\sum_{|\omega|=n} \exp \left(\sup \left(S_{\omega}(G)\right)\right)
$$

is subadditive, where $S_{\omega}(G)=\sum_{j=1}^{n} g^{\left(\omega_{j}\right)} \circ \phi_{\sigma^{j} \omega}$. Moreover

$$
\mathrm{P}(G)=\inf _{n \geq 1}\left\{\frac{1}{n} \log Z_{n}(G)\right\} .
$$

Now, a Borel probability measure $m_{G}$ is said to be $G$-conformal provided it is supported on the limit set $J$, for every Borel set $A \subset X$

$$
m_{G}\left(\phi_{\omega}(A)\right)=\int_{A} \exp \left(S_{\omega}(G)-\mathrm{P}(G)|\omega|\right) d m_{G}, \quad \text { for all } \omega \in I^{*}
$$

and $m_{G}\left(\phi_{\omega}(X) \cap \phi_{\tau}(X)\right)=0$ for all incomparable $\omega, \tau \in I^{*}$. In [6] and [16], (compare with [10]), the following is proved.

THEOREM 5.2. If $G$ is a summable Hölder family of functions, then there exists exactly one $G$-conformal measure $m_{G}$. In addition, there exists a unique shift-invariant Borel probability measure $\tilde{\mu}_{G}$ on $I^{\infty}$ such that the measure $\mu_{G}=\tilde{\mu}_{G} \circ \pi^{-1}$ on $J$ is absolutely continuous with respect to $m_{G}$. In addition, the Radon-Nikodym derivative $d \mu_{G} / d m_{G}$ is uniformly bounded away from zero and infinity.

The measure $\mu_{G}$ is called the Gibbs state of the Hölder family $G$. It follows from Theorem 5.2 and the statements above that we may consider the dynamical system $\left(f, \mu_{G}\right)$. Our aim is to verify that this dynamical system is loosely Markov. We start with the following. 
PROPOSITION 5.3. If $G$ is a summable Hölder family of functions with finite Lyapunov exponent $\chi_{\mu_{G}}$, then

$$
\lim _{r \rightarrow 0} \frac{\log \mu_{G}(B(x, r))}{\log r}=\frac{\mathrm{h}_{\mu_{G}}(f)}{\chi_{\mu_{G}}}=\mathrm{HD}\left(\mu_{G}\right) .
$$

for $\mu_{G}$-almost-everywhere $x \in J$.

PRoof. Define the function $\rho: I^{\infty} \rightarrow \mathbb{R}$ by the formula

$$
\rho(\omega)=\liminf _{r \rightarrow 0} \frac{\log m_{G}(B(x, r))}{\log r} .
$$

We shall show for every $\omega \in I^{\infty}$ that

$$
\rho(\sigma \omega) \geq \rho(\omega) .
$$

Indeed, using $G$-conformality of the measure $\mu_{G}$, we get for every $\omega \in I^{\infty}$ and every $r \in(0, \operatorname{dist}(X, \partial V))$ that

$$
\begin{aligned}
m_{G}\left(B\left(\pi(\omega),\left\|\phi_{\omega_{1}}^{\prime}\right\| r\right)\right) & \geq m_{G}\left(\phi_{\omega_{1}}(B(\pi(\sigma \omega), r))\right) \\
& =\int_{B(\pi(\sigma \omega), r)} \exp \left(g^{\left(\omega_{1}\right)}(\pi(\omega))-\mathrm{P}(G)\right) d m_{G} \\
& \geq e^{-\mathrm{P}(G)} \exp \left(\inf \left\{g^{\left(\omega_{1}\right)}\right\} m_{G}(B(\pi(\sigma \omega), r)) .\right.
\end{aligned}
$$

Hence, expression (5.2) follows. Since the measure $\tilde{\mu}_{G}$ is ergodic, it follows from Birkhoff's ergodic theorem that the function $\rho: I^{\infty} \rightarrow \mathbb{R}$ is constant $\tilde{\mu}_{G}$-almosteverywhere, say $\rho(\omega)=\hat{\rho}$ for some $\hat{\rho}>0$ and all $\omega \in Y \subset I^{\infty}$ with $\tilde{\mu}_{G}(Y)=1$. Set now

$$
\underline{\rho}(\omega)=\liminf _{r \rightarrow 0} \frac{\log \mu_{G}(B(x, r))}{\log r} .
$$

Since, by Theorem 5.2, the measures $\mu_{G}$ and $m_{G}$ are equivalent with bounded RadonNikodym derivatives, we have that $\rho=\rho$, and consequently, $\rho=\hat{\rho}$ for all $\omega \in Y$. Since $\mathrm{HD}\left(\mu_{\mathrm{G}}\right)=\operatorname{ess} \sup (\rho)$ and, in view of [10, Theorem 4.4.2] (compare with [16, Theorem 3.1]), we have $\mathrm{HD}\left(\mu_{G}\right)=\mathrm{h}_{\mu_{G}}(f) / \chi_{\mu_{G}}$, we therefore conclude that

$$
\liminf _{r \rightarrow 0} \frac{\log \mu_{G}(B(x, r))}{\log r}=\frac{\mathbf{h}_{\mu_{G}}(f)}{\chi_{\mu_{G}}}=\operatorname{HD}\left(\mu_{G}\right) .
$$

for all $\omega \in Y$. Since it was demonstrated in the proof of [10, Theorem 4.4.2] (compare with Theorem 3.1) that

$$
\limsup _{r \rightarrow 0} \frac{\log \mu_{G}(B(x, r))}{\log r} \leq \frac{\mathbf{h}_{\mu_{G}}(f)}{\chi_{\mu_{G}}}
$$


for $\tilde{\mu}_{G}$-almost-everywhere $\omega \in I^{\infty}$, we therefore conclude that

$$
\lim _{r \rightarrow 0} \frac{\log \mu_{G}(B(x, r))}{\log r}=\frac{\mathrm{h}_{\mu_{G}}(f)}{\chi_{\mu_{G}}}=\operatorname{HD}\left(\mu_{G}\right)
$$

for $\tilde{\mu}_{G}$-almost-everywhere $\omega \in I^{\infty}$. The proof is complete.

The iterated function system $\left\{\phi_{i}\right\}_{i \in I}$ is said to satisfy the strong open set condition if $J \cap \operatorname{Int}_{\mathbb{R}^{d}}(X) \neq \emptyset$. In the case when the alphabet $I$ is finite, then the strong open set condition is always satisfied (see [11]) perhaps with a different seed set $X$. The main result of this section is the following.

THEOREM 5.4. Suppose that $\left\{\phi_{i}\right\}_{i \in I}$ is a conformal iterated function system satisfying the strong open set condition. Suppose also that $G$ is a summable Hölder family of functions with finite Lyapunov exponent $\chi_{\mu_{G}}$. If $\left(f, \mu_{G}\right)$ is the corresponding dynamical system, then for $\mu_{G}$-almost-everywhere $z \in J$, the number $R_{\mu_{G}}(z)$ exists and moreover $R_{\mu_{G}}(z)=\mathrm{HD}\left(\mu_{G}\right)=\mathrm{h}_{\mu_{G}}(f) / \chi_{\mu_{G}}$.

ProOF. We shall verify that the dynamical system $\left(f, \mu_{G}\right)$ is loosely Markov. Condition (a) follows immediately from [10, Theorem 2.4.6] and the relation $L_{\phi}(1)(\omega)=$ $\tilde{L}_{\phi}(1)(\pi \omega)$ established in [7, page 128]. In order to check condition (b) put $\chi=\chi_{\mu_{G}}$. Let $W_{1} \subset I^{\infty}$ be the set of all sequences $\omega \in I^{\infty}$ such that

$$
\lim _{n \rightarrow \infty} \frac{1}{n} \log \left|\phi_{\omega_{i}}^{\prime}\left(\pi\left(\sigma^{i+1} \omega\right)\right)\right|=-\chi .
$$

By Birkhoff's ergodic theorem, $\tilde{\mu}_{G}\left(W_{1}\right)=1$. The strong open set condition implies the existence of a finite word $\eta \in I^{*}$ such that $\phi_{\eta}(X) \subset$ Int $X$. Let $r=$ $\operatorname{dist}\left(\phi_{\eta}(X), \partial X\right)$. It follows from Birkhoff's ergodic theorem that there exists a Borel set $W_{2} \subset W_{1}$ such that $\tilde{\mu}_{G}\left(W_{2}\right)=1$, and for every $\omega \in W_{2}$

$$
\lim _{k \rightarrow \infty} \frac{n_{k+1}(\omega)}{n_{k}(\omega)}=1
$$

where $\left\{n_{k}(\omega)\right\}_{k=1}^{\infty}$ is the infinite sequence of all consecutive integers $n \geq 1$ such that $\sigma^{n}(\omega) \in\{\eta\} \times I^{\infty}$. In particular $\mu_{G}\left(\pi\left(W_{2}\right)\right)=1$. The family $\alpha=\left\{\phi_{i}(X)\right\}_{i \in I}$. is a partition of $J$. Fix $x \in \pi\left(W_{2}\right), x=\pi(\omega)$, where $\omega \in W_{2}$. Using the bounded distortion property and expressions (5.3) and (5.4), we see that for all $n \geq 1$ large enough, $\left|\phi_{\left.\omega\right|_{n}}^{\prime}(x)\right| \geq r^{-1} \exp (-2 \chi n)$ and $n_{k+1} \leq 2 n_{k}$, where $k \geq 1$ is uniquely determined by the requirement that $n_{k} \leq n<n_{k+1}$. We then have that

$$
\begin{aligned}
\alpha_{n}(x) & =\phi_{\left.\omega\right|_{n}}(X) \supset \phi_{\left.\omega\right|_{n_{k+1}}}(X) \\
& \supset \phi_{\left.\omega\right|_{n_{k+1}}}\left(B\left(\pi\left(\sigma^{n_{k+1}} \omega, r\right)\right)\right) \supset B\left(\pi(\omega), \exp \left(-2 \chi n_{k+1}\right)\right) \\
& \supset B\left(\pi(\omega), \exp \left(-4 \chi n_{k}\right)\right) \supset B(\pi(\omega), \exp (-4 \chi n)) .
\end{aligned}
$$


So, condition (b) of the definition of loosely Markov systems is also satisfied. Condition (c) follows immediately from Proposition 5.3. Thus the proof is completed by applying Theorem 2.1 along with Proposition 5.3.

\section{References}

[1] L. Barreira and B. Saussol, 'Hausdorff dimensions of measures via Poincaré recurrence', Comm. Math. Phys. 219 (2001), 443-463.

[2] — - 'Product structure of Poincaré recurrence', Ergodic Theory Dynam. Systems 22 (2002), $33-61$.

[3] R. Benedetti and C. Petronio, Lectures on hyperbolic geometry (Springer, Berlin, 1992).

[4] M. Boshernitzan, 'Quantitative recurrence results', Invent. Math. 113 (1993), 617-631.

[5] M. Denker and M. Urbański, 'Ergodic theory of equilibrium states for rational maps', Nonlinearity 4 (1991), 103-134.

[6] P. Hanus, D. Mauldin and M. Urbański, 'Thermodynamic formalism and multifractal analysis of conformal infinite iterated function systems', Acta Math. Hungarica 96 (2002), 27-98.

[7] P. Hanus and M. Urbański, 'A new class of positive recurrent functions', in: Geometry and topology in dynamics, Contemporary Mathematics Series of the AMS 246 (Amer. Math. Soc., Providence, RI, 1999) pp. 123-136.

[8] N. Haydn, 'Convergence of the transfer operator for rational maps', Ergodic Theory Dynam. Systems 19 (1999), 657-669.

[9] R. D. Mauldin and M. Urbański, 'Dimensions and measures in infinite iterated function systems', Proc. London Math. Soc. (3) 73 (1996), 105-154.

[10] - Graph directed Markov systems: geometry and dynamics of limit sets (Cambridge Univ. Press, 2003).

[11] Y. Peres, M. Rams, K. Simon and B. Solomyak, 'Equivalence of positive hausdorff measure and the open set condition for self-conformal sets', Proc. Amer. Math. Soc. 129 (2001), 2689-2699.

[12] F. Przytycki and M. Urbański, Fractals in the plane. The ergodic theory methods (Cambridge Univ. Press, to appear).

[13] P.Walters, An introduction to ergodic theory (Springer, 1982).

[14] B. Saussol, S. Troubetzkoy and S. Vaienti, 'No small return', in preparation.

[15] M. Urbański, 'Thermodynamic formalism and multifractal analysis for hyperbolic exponential functions', Preprint, 2005.

[16] — - 'Hausdorff measures versus equilibrium states of conformal infinite iterated function systems', Period. Math. Hungar. 37 (1998), 153-205.

[17] M. Urbański and A. Zdunik, 'Maximizing measures on metrizable non-compact spaces', Preprint, 2004, to appear in Proc. Edinb. Math. Sac.

Department of Mathematics

University of North Texas

P.O. Box 311430

Denton TX 76203-1430

USA

e-mail: urbanski@unt.edu 